\title{
Very Low Pressure Pyrolysis of Phenylacetic Acid
}

\author{
A. J. Colussi, V. T. Amorebieta and M. A. Grela \\ Department of Chemistry, University of Mar del Plata, (7600) Mar del Plata, Argentina
}

A kinetic study of the very low pressure thermolysis of phenylacetic acid above $900 \mathrm{~K}$ reveals that its decompo-
sition occurs in a concerted manner, yielding toluene and carbon dioxide, probably via a four-centre transition state [reaction (1)]

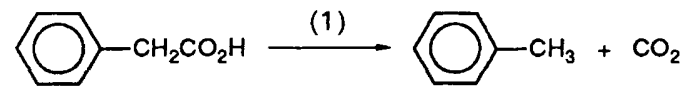

with $\log \left[k_{\infty}(1) / s^{-1}\right]=[(13 \pm 0.3)-(12200 \pm 500)] / T$.

Recent reviews on the thermochemistry of organic radicals in the gas phase, ${ }^{1,2}$ have pointed out the necessity of a reassessment of the heat of formation of the hydroxycarbonyl radical and the corresponding bond-dissociation energy $E_{\mathrm{d}}$ in formic acid. Based on a re-evaluation of the early work of Back and Sehon, ${ }^{3}$ McMillen and Golden, ${ }^{1}$ have recommended $\Delta_{\mathrm{f}} H\left({ }^{\circ} \mathrm{CO}_{2} \mathrm{H}\right)=-209.2 \mathrm{~kJ} \mathrm{~mol}^{-1}$; however, standard correlations between $\mathrm{C}-\mathrm{H}$ stretching frequencies and bonddissociation energies indicate a stronger bond. ${ }^{2,4}$

In order to bring some progress to this issue, we decided to reinvestigate the thermolysis of phenylacetic acid at very low pressures, conditions that would effectively isolate the primary step:

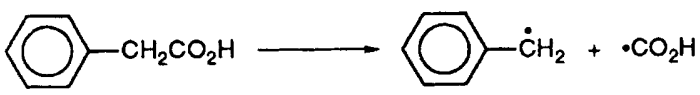

However, we were faced with an alternative lower energy path to the single-bond-fission process, which yields toluene and carbon dioxide instead of the expected stabilized benzyl radicals.

\section{Experimental}

As described elsewhere, ${ }^{5,6}$ in very low pressure experiments the reactants become thermalized in a few gas-wall collisions and subsequently undergo unimolecular decomposition in the fall-off region. The resulting fragments leave the reactor before engaging in secondary bimolecular reactions. This technique has proved to be particularly suitable for the study of simple bond-fission reactions where radicals are formed. In order to preserve the identity of these reactive species, molecular-beam sampling is highly desirable, as radicals are known to react readily upon collision with cold metal surfaces.

In our experiments, a steady flow of reactant vapour from a solid sample contained in a temperature-controlled reservoir, was admitted to a silica flow reactor through a capillary restriction. To prevent condensation the entire flow system was electrically heated at least $10 \mathrm{~K}$ above the sample temperature.

The reactor was encased in a stainless-steel block and heated by means of two semi-cylindrical heating units. It was characterized by an escape rate constant $k_{\mathrm{eM}} / \mathrm{s}^{-1}=$ $0.214(T / M)^{1 / 2}$ and a gas-wall collision frequency $w / \mathrm{s}^{-1}=$ $4.53 \times 10^{3}(T / M)^{1 / 2}(M$ in $u)$, which determines the degree of fall-off.

The temperature was measured by means of two calibrated $\mathrm{Pt}-10 \% \mathrm{Rh} / \mathrm{Pt}$ thermocouples, located at the top and at the bottom of the reactor. Gradients along it were less than $\pm 1 \mathrm{~K}$
The effusing gases coming from the reactor, were admitted to the differential pumping chamber of a modulated-beam mass spectrometer (EMBA II, Extrel) where a molecular beam was created. Then, they were admitted to the main chamber through a $1 \mathrm{~mm}$ collimating aperture where they were modulated, ionized by electron impact and analysed with a quadrupole mass filter. The output was finally processed by a lock-in amplifier.

Unimolecular rate constants, $k_{\text {uni }}$, were calculated from the fraction of reactant decomposed at each temperature, $f$, by the expression:

$$
k_{\text {uni }}=k_{\mathrm{eM}} f /(1-f)
$$

Flow rates, determined by Knudsen's method, were kept between $5 \times 10^{13}$ and $5 \times 10^{14}$ molecules $\mathrm{s}^{-1}$. Under these conditions, even the fastest bimolecular reactions become much slower than the escape rate of species from the reactor. ${ }^{6}$

Phenylacetic acid (Aldrich, 99\%) was used as received.

\section{Results}

\section{Kinetic data}

Phenylacetic acid was thermolysed between 870 and $1020 \mathrm{~K}$. The extent of reaction, $f$, was followed by the decay of molecular ion signal intensity at $m / z=136$. Systematic experimental errors in $k_{\mathrm{uni}}$ arising from reactor parameters were prevented. This was demonstrated by the fact that the rates of decomposition of ethylbenzene in our set-up were in excellent agreement with literature reports and thermodynamic data. ${ }^{7.8}$

The results were quite reproducible and independent of a ten-fold variation in flow rates (see Fig. 1).

\section{Product Analysis}

Mass spectral patterns were obtained at $15 \mathrm{eV}$ to minimize ion fragmentation. The room-temperature mass spectrum of phenylacetic acid contains a major fragment at $m / z=91$ besides the molecular ion at $m / z=136$. Above $900 \mathrm{~K}$, this ion intensity decreased markedly and two new ion signals at $m / z=44$ and 92 appeared in the spectrum. In addition, phase shifts ${ }^{9}$ confirmed them as genuine products identified as carbon dioxide and toluene. The possibibility that the peak of mass 92 results from hydrogen abstraction by the benzyl radical that would be formed by bond fission [reaction (2)] can be discarded. Here, molecular-beam sampling plays an important role, as it preserves the identity of the radicals formed, preventing $\mathrm{H}$ abstraction from metal surfaces in the ionization chamber. As a test, we verified that our experimental set-up allows us to detect methyl and benzyl radicals 


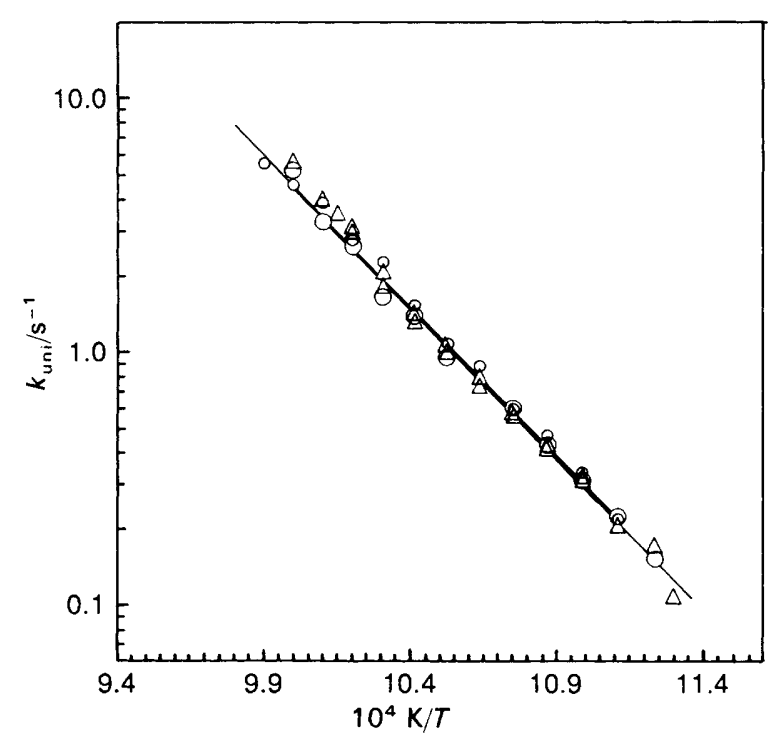

Fig. 1 Arrhenius plot of the measured rate constants. Phenylacetic flow rates: $\triangle, 5 \times 10^{13}$ and $O, 5 \times 10^{14}$ molecule $^{-1}$. Solid line is derived from Troe's fit (see text)

formed in ethylbenzene thermolysis. ${ }^{8}$ A ten-fold variation of flow rates did not modify either the spectral pattern of the effusing reaction mixtures or the extent of decomposition. Therefore, it also eliminates the possibility of secondary radical-radical or radical-molecule reactions as intermediate steps. Moreover, we have also been able to establish that for each molecule of phenylacetic acid decomposed an equivalent amount of $\mathrm{CO}_{2}$ is formed, since the same values for $k_{\text {uni }}$ were obtained when the extent of decomposition was evaluated from measurements of the signal at $m / z=44$.

\section{Discussion}

Under present experimental conditions the collision frequencies are insufficient to maintain an equilibrium popu- lation of reacting states; therefore, the measured values of $k_{\text {uni }}$ correspond to the fall-off region. In these conditions, the high-pressure-limit rate constant can be derived from experimental data by means of any of the unimolecular rate theories once the structure of the transition state has been assigned. ${ }^{4,10}$

From the experimental evidence described in the previous section, we concluded that carbon dioxide and toluene are actually the products of a concerted reaction. Accordingly, we postulate the four-centre transition state depicted below:

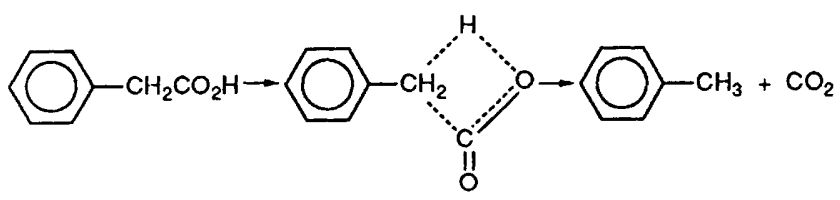

Following the classical work of O'Neal and Benson, ${ }^{11}$ we made a detailed analysis of the structural evolutions associated with formation of the transition state. Table 1 summarizes the individual contributions made by each mode that changes during reaction to give $\Delta S^{\ddagger}$. Note that the estimated value of $A_{\infty}(1)=10^{13} \mathrm{~s}^{-1}$ arises mainly from the replacement of internal rotations by low-frequency torsions in the transition state and it is completely in accord with the corresponding values reported for other four-centre concerted processes. ${ }^{10,12,13}$

The degree of fall-off was then evaluated by the method of Troe. ${ }^{14,15}$ He has developed a simple formulation in which the intermediate fall-off range is expressed as a transition between the low-pressure range, where collisional energy transfer is rate determining, and the limiting high-pressure range, where the rate-determining processes are intramolecular. It basically consists of a modified form of the Lindemann-Hinshelwood equation:

$$
k_{\text {uni }} / k_{\infty}=k_{0} /\left[k_{\infty}\left(1+k_{0} / k_{\infty}\right)\right]=F_{\mathrm{LH}}
$$

by the incorporation of two 'broadening' terms:

$$
k_{\mathrm{uni}} / k_{\infty}=F_{\mathrm{LH}} F_{\mathrm{sc}} F_{\mathrm{wc}}
$$

\begin{tabular}{|c|c|c|c|c|c|c|}
\hline \multicolumn{3}{|c|}{ ground state } & \multicolumn{4}{|c|}{ transition state ${ }^{a}$} \\
\hline & frequency $/ \mathrm{cm}^{-1}$ & $S$ & & frequency $/ \mathrm{cm}^{-1}$ & $s$ & $\Delta S$ \\
\hline$c-0$ & 1200 & 1.13 & $c-0$ & 1400 & 0.90 & -0.23 \\
\hline $\mathrm{O}-\mathrm{H}$ & 3100 & 0.13 & $\mathrm{O} \cdot \mathrm{H}$ & 2200 & 0.36 & 0.23 \\
\hline$c-c$ & 1000 & 1.43 & $\begin{array}{l}\text { reaction } \\
\text { coordinate }\end{array}$ & - & - & -1.43 \\
\hline & 400 & 3.11 & $\mathrm{c}^{\cdot}$ & 280 & 3.80 & 0.69 \\
\hline$c^{-C}$ & 420 & 3.20 & $c$ & 250 & 4.00 & 1.00 \\
\hline & 1150 & 1.20 & $\mathrm{H}^{\bullet}$ & 800 & 1.81 & 0.61 \\
\hline $\mathrm{H}^{\prime}$ & 1150 & 1.20 & $\mathrm{H}^{-}$ & 750 & 1.93 & 0.73 \\
\hline internal rotation & $V_{0} / \mathrm{kJ} \mathrm{mol}^{-1}$ & $s$ & torsion & frequency $/ \mathrm{cm}^{-1}$ & $s$ & $\Delta S$ \\
\hline $\mathrm{PhCH}_{2} \mathrm{C} / \mathrm{OH}$ & 50.2 & $3.0^{b}$ & $\mathrm{H}_{2} \mathrm{C}^{\mathrm{O}}$ & 300 & 3.67 & -0.67 \\
\hline $\mathrm{PhCH}_{2}\left\{\mathrm{C}_{\mathrm{OH}}^{\prime \prime}\right.$ & 8.4 & $7.8^{b}$ & $\mathrm{H}_{2} \mathrm{C}=\mathrm{C}_{=0}^{\prime O}$ & 350 & 3.37 & $\Delta S_{i}^{\ddagger}=\frac{-4.43}{-3.50}$ \\
\hline
\end{tabular}

Table 1 Structural assignment for the four-centre transition state

$T=10^{3} \mathrm{~K}$; entropies in cal mol ${ }^{-1} \mathrm{~K}^{-1}$. ${ }^{a}$ see ref. $11 .{ }^{b}$ Reported entropies are free rotor values corrected by rotational barriers. ${ }^{c} \log \left(A_{\infty} / \mathrm{s}^{-1}\right)=\log (e k T / h)+\Delta S_{\mathfrak{i}}^{\ddagger} / 4.575=13.0$. 
where $F_{\mathrm{sc}}$ corrects for the energy dependence of the microcanonical dissociation rate constants for the adduct and $F_{\mathrm{wc}}$ corrects for weak collision effects. Both factors are a function of $k_{0} / k_{\infty}$. The limiting low-pressure pseudo-first-order rate constant, $k_{0}$, has been expressed in a factorized form: ${ }^{15}$

$$
\begin{aligned}
k_{0}= & {\left[\omega \beta_{\omega} \rho_{\mathrm{vib}, \mathrm{h}}\left(E_{0}\right) / k T \exp \left(-E_{0} / k T\right)\right.} \\
& \left.\times F_{\text {anh }} F_{\mathrm{E}} F_{\text {ext rot }} F_{\text {int rot }} F_{\text {corr }}\right] Q_{\mathrm{vib}}^{-1}
\end{aligned}
$$

where $\omega$ represents the collision frequency and $\beta_{\omega}$ its efficiency. Other symbols adhere fully to Troe's notation. The input data are shown in Appendix A.

The calculation shows that the measured rate constants are not very far from the high-pressure limit $\left(k_{\text {uni }} / k_{\infty}\right.$ varies with $T$ between 0.77 and 0.86 ). The best fit leads to:

$$
\log \left[k_{\infty}(1) / \mathrm{s}^{-1}\right]=[(13 \pm 0.3)-(12200 \pm 500)] / T
$$

The quoted errors arise mainly from an uncertainty of $c a$. a factor of two in $A_{\infty}(1),{ }^{10,12}$ the lack of definitive information about the value of $\beta_{\omega}, \dagger$ and from the evaluation of the extent of fall-off itself.

Expression (I) can be contrasted directly with the early study of Back and Sehon, who reported $\log k=12.9-12000 / T$ for phenylacetic acid disappearance. ${ }^{3}$ Considering that their experiments were performed directly at infinite pressure, the agreement is highly satisfactory. However, these authors had ascribed the measured rates to reaction (2), even when there was no experimental evidence to support this assumption. In fact, as it has been previously pointed out by Benson and O'Neal, the observed pre-exponential factor seems inadequately low for a simple bond-fission reaction and is actually outside the accepted error limits. ${ }^{10,12,18,19}$ By analogy with similar processes, a reasonable estimation would probably fix $\log A_{\infty}(2)=15 \pm 0.3 .^{12}$ There is no way to fit their experimental points with this constraint over all the range of temperatures studied. On the other hand, confining the fit to a small temperature range centred at $940 \mathrm{~K}$, one obtains $E_{\infty}(2)=270 \pm 16 \mathrm{~kJ} \mathrm{~mol}^{-1}$. This result, combined with well established data leads to a value of $334.7 \pm 16 \mathrm{~kJ} \mathrm{~mol}^{-1}$ for the bond dissociation energy in formic acid, $\ddagger$ which seems too low using thermochemical arguments. ${ }^{4,12}$

In conclusion, it can be concluded confidently on the basis of experimental and thermochemical evidence that the unimolecular decomposition of phenylacetic acid does not involve a simple bond fission as previously assumed and instead it yields toluene and carbon dioxide concertedly.

\section{Appendix \\ Input Data for Troe's calculations at $950 \mathrm{~K}$}

Ground-state Frequencies $/ \mathrm{cm}^{-1}$ a

$3550,3100,3050$ (4), 2950 (2), 1750, 1600 (2), 1450 (4), 1300 (3), 1200, $1150(4), 1050(2), 1000(2), 950(3), 900,850(2), 750(2)$, $700,600(3), 500,400(3), 300,250,200$.

Transition-state Frequencies $/ \mathrm{cm}^{-1} b$

3550,3050 (4), 2950 (2), 2200, 1750, 1600 (2), 1450 (4), 1400, $1300(3), 1150(2), 1050(2), 1000(2), 950(3), 900,850(2), 800$, 750 (3), 700, 600 (3), 500, 400 (3), 300, 250, 200.

$$
E_{0}=E_{\infty}=\text { fitting parameter }
$$

$$
\begin{array}{r}
\rho_{\text {vib }, \mathrm{h}}\left(E_{0}\right)=5.816 \times 10^{16} \mathrm{~mol} \mathrm{kcal}^{-1} c \\
\beta_{\omega}{ }^{d}=1 \\
\left(I^{+} / I\right)^{e}=1
\end{array}
$$

${ }^{a}$ Estimated by adjusting generic local mode frequencies to overall vibrational heat capacity. ${ }^{b}$ See Table $1 .{ }^{c}$ Density of states at $E_{0}=230.0 \mathrm{~kJ} \mathrm{~mol}^{-1}$, calculated from the WhittenRabinovitch equation. ${ }^{10}{ }^{d}$ See ref. $16 .{ }^{e}$ Ratio of overall moments of inertia of the activated complex to that of the ground-state molecule.

\section{References}

1 D. F. McMillen and D. M. Golden, Annu. Rev. Phys. Chem., 1982, 33, 493.

2 A. J. Colussi, Chemical Kinetics of Small Radicals, ed. Z. Alfassi, Uniscience Series, CRC Press, Boca Raton, FL, 1988, ch. 3.

3 A. H. Sehon and M. H. Back, Can. J. Chem., 1960, 38, 1261.

4 D. C. McKean, J. L. Duncan and L. Batt, Spectrochim. Acta, Part A, 1973, 29, 1037.

5 M. A. Grela and A. J. Colussi, Int. J. Chem. Kinet., 1985, 17, 277.

6 D. M. Golden, G. M. Spokes and S. W. Benson, Angew. Chem. Int. Ed. Engl. 1973, 12, 534.

7 H. Hipler and J. Troe, J. Phys. Chem., 1990, 94, 3803.

8 D. A. Robaugh and S. E. Stein, Int. J. Chem. Kinet., 1981, 13, 445.

9 V. T. Amorebieta and A. J. Colussi, J. Phys. Chem., 1982, 86, 2760.

10 P. J. Robinson and K. A. Holbrook, Unimolecular Reactions Wiley, New York, 1974.

11 H E. O'Neal and S. W. Benson, J. Phys. Chem., 1967, 71, 2903.

12 S. W. Benson, Thermochemical Kinetics, Wiley, New York, 2nd edn., 1976

13 E. Ghibaudi and A. J. Colussi, Int. J. Chem. Kinet., 1984, 16, 1575 .

14 M. A. Grela and A. J. Colussi, Int. J. Chem. Kinet., 1988, 20, 713.

15 J. Troe, J. Phys. Chem., 1979, 83, 114

16 R. G. Gilbert, Int. J. Chem. Kinet., 1982, 14, 447

17 V. T. Amorebieta, and A. J. Colussi, J. Phys. Chem., 1982, 86, 3058 .

18 H. E. O'Neal and S. W. Benson, Kinetic Data on Gas-phase Unimolecular Reactions, Washington, DC, 1970, Natl. Ref. Data Serv. NSRDS-NBS 21, US.

19 H. E. O'Neal and S. W. Benson, Free Radicals, ed. J. K. Kochi, Wiley, New York, 1973, vol. II. 